\title{
Case study on the effect of percutaneous needle electrolysis in mammary fistulae
}

\author{
García Vidal J.A. ${ }^{1}$ Berna Serna J.D. ${ }^{2} \quad$ Escolar Reina P. ${ }^{1} \quad$ Berna Mestre J.D. ${ }^{3}$ \\ 1 Department of Physiotherapy, Universidad de Murcia, Murcia, Spain \\ ${ }^{2}$ Servicio de Radiología, Hospital Universitario Virgen de la Arrixaca, \\ Murcia, Spain \\ ${ }^{3}$ Radiology Service, Hospital Universitario Virgen de la Arrixaca, \\ Murcia, Spain \\ Rev Fisioter Invasiva 2019;2:89-90.
}

\begin{abstract}
Background and Aims The mammary fistula (MF) was described by Zuska (1951) and is commonly associated with a non-lactational subareolar abscess, which is a particularly difficult condition due to the intense discomfort and tendency for recurrence. It is estimated to affect non-breastfeeding women aged between 20 and 50 years, and is responsible for $1-2 \%$ of all the symptomatic breast affections. Recent studies have highlighted a dermatological pathogenesis, confirming the existence of chronic inflammation of the pilosebaceous follicles and the surrounding tissue. To date, the first choice of treatment in initial phases has been the drainage and aspiration of the MF combined with the prescription of wide spectrum antibiotics. Other authors have defended the use of corticosteroid infiltrations (triamcinolone). However, these solutions are far from being definitive, as the recurrence rate is quite high, especially when purulent content exists. Percutaneous needle electrolysis (PNE) has demonstrated its effectiveness in pathologies which are accompanied with chronic inflammation, such as patellar and Achilles tendinopathy, subacromial syndromes and epicondyalgia. The aim of this study was to examine the effects produced by the application of PNE in 3 patients with MF.

Material and Methods Three patients with relapsing MF were recruited who fulfilled the inclusion criteria. All the exams and interventions were performed under ultrasound guidance by an expert radiologist. Two interventions were performed, separated 14 days and with a follow up of 15, 30, and 60 days after the application. Prior to treatment, an exhaustive ultrasound exam was performed (Phillips $₫$ ultrasound with a linear probe $14-5 \mathrm{MHz}$ ) in the intervention area. After the corresponding antiseptic measures were performed, the abscess was anesthetized and drained (1-2 mL mepivacaine and physiological saline), using an Introcan ${ }^{\circledR}$ Safety needle of $14 \mathrm{G}$. For the application of PNE, the same needle was used, connected to the Physio Invasiva device

Keywords

- mammary fistula

- breast

- percutaneous electrolysis

- chronic inflammation (Grupo PRIM $®$ ), with a dose of 5 impacts of $5 \mathrm{~mA}$ during 5 seconds.

Results All the patients experienced an improvement in their clinical status. The ultrasound controls of the patients displayed a decrease of the abscess and changes in the echogenicity of the same after 30 days: Two of these reported a disappearance of suppuration episodes, whereas one experienced a decrease of the same, both in quantity as well as frequency.
\end{abstract}

DOI https://doi.org/ 10.1055/s-0039-3401869. ISSN 2386-4591.
Copyright ( 2019 by Thieme Revinter Publicações Ltda, Rio de Janeiro, Brazil 
90 Abstract 23

Conclusion PNE can be an effective therapeutic option in the treatment of mammary fistulae. Further studies are required along these lines to verify and complete our preliminary findings. 